\title{
Functional changes in neocortical activity in Huntington's disease model mice: an in vivo intracellular study
}

\author{
Edward A. Stern ${ }^{1,2 *}$ \\ Brain Research Center, Bar-Ilan University, Ramat Gan, Israel \\ 'Department of Neurology, MassGeneral Institute for Neurodegenerative Disease, Massachusetts General Hospital, Harvard Medical School, Charlestown, MA, USA
}

Edited by:

Elizabeth Abercrombie, Rutgers-

Newark: The State University of New

Jersey, USA

\section{Reviewed by:}

Michael S. Levine, Brain Research Institute, USA

George V. Rebec, Indiana University, USA

\section{${ }^{*}$ Correspondence:}

Edward A. Stern, Brain Research Center, Bar-llan University, Ramat Gan 52900, Israel.

e-mail: sterned@mail.biu.ac.il
Studies of animal models of Huntington's disease (HD) have revealed that neocortical and neostriatal neurons of these animals in vitro exhibit a number of morphological and physiological changes, including increased input resistance and changes in neocortical synaptic inputs. We measured the functional effects of polyglutamate accumulation in neocortical neurons in R6/2 mice (8-14 weeks of age) and their age-matched non-transgenic littermates using in vivo intracellular recordings. All neurons showed spontaneous membrane potential fluctuations. The current/voltage and the firing properties of the HD neocortical neurons were significantly altered, especially in the physiologically relevant current range around and below threshold. As a result, membrane potential transitions from the Down state to Up state were evoked with smaller currents in HD neocortical neurons than in controls. The excitation-to-frequency curves of the $\mathrm{HD}$ mice were significantly steeper than those of controls, indicating a smaller input-output dynamic range for these neurons. Increased likelihood of Down to Up state transitions could cause pathological recruitment of corticostriatal assemblies by increasing correlated neuronal activity. We measured coherence of the in vivo intracellular recordings with simultaneously recorded electrocorticograms. We found that the peak of the coherence at $<5 \mathrm{~Hz}$ was significantly smaller in the HD animals, indicating that the amount of coherence in the state transitions of single neurons is less correlated with global activity than non-transgenic controls. We propose that decreased correlation of neocortical inputs may be a major physiological cause underlying the errors in sensorimotor pattern generation in HD.

Keywords: Huntington's disease, cortex, striatum, R6/2, intracellular recording, in vivo

\section{INTRODUCTION}

The corticostriatal pathway is one of the two major inputs to the basal ganglia, containing inputs from virtually every neocortical area. The role of this pathway in sensorimotor and cognitive behavior is only beginning to be unraveled. One key to understanding the corticostriatal neural mechanisms underlying behavior is to study the changes in neocortical activity when it is disrupted by a disease. Huntington's disease (HD) is a neurodegenerative disease primarily affecting the projection neurons in the neocortex and neostriatum (Vonsattel et al., 1985). Symptoms of HD include involuntary movements, but also include sensory and cognitive disruptions, as would be expected when the neuropathology affects large telencephalic areas. HD is characterized by accumulation of abnormal protein (huntingtin) which aggregates in the nucleus and cytoplasm of neocortical pyramidal neurons and neostriatal medium spiny neurons (Davies et al., 1997; DiFiglia et al., 1997). The functional changes in neocortical and neostriatal activity underlying the symptoms of $\mathrm{HD}$ begin prior to neurodegeneration, and the mechanisms underlying the changes are poorly understood. Following the discovery of the HD gene (HDCRG, 1993), a number of animal models of the disease have been used to characterize the cellular changes in neocortical neurons and neostriatal neurons prior to degeneration. One of the best known and studied HD transgenic models is the R6/2 line (Mangiarini et al., 1996, 1997; Davies et al., 1997; Bates et al., 1998). In these animals, neocortical and neostriatal projection neurons develop intranuclear inclusions with a well-established timeline in which all neurons have the inclusions by 8 weeks of age. The animals show behavioral dysfunctions, and die at about 12-14 weeks of age. The relatively short lifespan and fast development of pathology makes these mice ideal for study of pathophysiology, as all of the projection neurons develop inclusions, and no notable neuronal death occurs.

A number of in vitro studies have shown altered neurophysiological and neuroanatomical properties of neocortical and neostriatal neurons in the R6/2 mice (Cepeda et al., 2001, 2003a, 2004; Klapstein et al., 2001; Laforet et al., 2001; Levine et al., 2004; Ariano et al., 2005; Andre et al., 2006; Cepeda and Levine, 2006). These studies show significant alterations in neocortical and neostriatal electrophysiological properties including both intrinsic conductances and synaptic properties. Changes in the properties of neostriatal neurons include decreased resting membrane potential, increased input resistance, and pruning of dendritic branching and spine patterns. Physiological changes also include reductions in synaptic currents evoked by stimulation of neocortical white matter (Cepeda et al., 2003a).

The changes in neuronal properties measured in vitro are crucial to understanding the effects of the neuropathology on cellular activity. However, in order to understand the changes in the corticostriatal network properties caused by HD pathology, it is necessary to measure the activity of these neurons in the intact animal. 
In vivo activity of neocortical neurons has been well characterized in rats (Steriade et al., 1993; Cowan and Wilson, 1994; Timofeev et al., 2000). The membrane potential dynamics of these neurons include spontaneous membrane potential transitions between the quiescent resting membrane potential (Down state) and a depolarized Up state, from which action potential arise. The mechanisms of the spontaneous membrane potential activity in neocortical neurons have been described in detail (Stern et al., 1997; Sachdev et al., 2004). These studies show that the Up states arise from barrages of neocortical synaptic activity interacting with non-linear membrane potential conductances. These barrages of synaptic activity are not present in acute slices, therefore, Up states are not seen in these preparations. To measure the functional effects of HD pathology on the activity of the neocortical network, we measured spontaneous activity and input-output relationships of neocortical neurons in the R6/2 mouse model of HD at time points when all neocortical pyramidal neurons show polyglutamine inclusions, and compared the results to those in age-matched non-transgenic littermates.

\section{MATERIALS AND METHODS}

Experiments were approved by the Institutional Animal Care and Use Committees of Massachusetts General Hospital and Bar-Ilan University in accordance with regulations approved by the US National Institutes of Health and the Israeli Ministry of Health.

\section{SURGERY}

R6/2 mice and non-transgenic littermate controls were used for this study (Mangiarini et al., 1996, 1997; Davies et al., 1997; Bates et al., 1998). The population had a CAG length of $\sim 160$ repeat expansions. The ages of the mice ranged from 8 to 14 weeks. Mice were anesthetized with ketamine/xylazine $(150 / 12 \mathrm{mg} / \mathrm{kg})$ and placed in a custom-built stereotaxic device. Temperature was maintained at $37^{\circ} \mathrm{C}$. The scalp was removed and the skull cleaned of tissue. Small holes $(1 \mathrm{~mm})$ were drilled for electrocorticogram (ECoG) electrodes over cortex and cerebellum. ECoG electrodes consisted of teflon-insulated silver wire with $1 \mathrm{~mm}$ insulation removed placed between dura and skull and cemented in place. ECoG was monitored continuously from the time of electrode placement to monitor depth of anesthesia. A $\sim 4 \mathrm{~mm} \times 4 \mathrm{~mm}$ craniotomy was opened over frontal cortex extending caudally to bregma. A cisternal drain was opened to reduce brain pulsations.

\section{RECORDING}

Recording electrodes (1.5 mm o.d. glass (A-M Systems); resistance 30-80 M $\Omega$; filled with $1 \mathrm{M}$ potassium acetate (Sigma) were lowered into agranular cortex. Following insertion of the recording electrode, the brain was covered with either $4 \%$ agarose or low-temperature melting point wax. Recordings were made under lighter conditions of anesthesia than are commonly used, as the R6/2 line and non-transgenic cohorts are more sensitive to anesthesia than are other mouse lines. The criteria used were evidence of slow-wave activity in the ECoG and lack of response to foot-pinch.

When a neuron was impaled, several minutes of spontaneous activity were recorded using a high-impedance amplifier with active bridge circuitry (IR-283; Cygnus Technology) Data were directly acquired via A/D board (National Instruments) using custom software at $4-10 \mathrm{kHz}$. For current-voltage and excitation-to-frequency curves, $200 \mathrm{~ms}$ current pulses were injected via the recording electrodes. Data were acquired using Igor Pro (Wavemetrics) or Spike 2/Signal (Cambridge) software. Analysis was performed using Igor Pro, Matlab (MathWorks), and SAS (SAS Institute) software.

\section{RESULTS}

All recorded neurons showed spontaneous membrane potential fluctuations. Figures 1A,B show representative recordings from non-transgenic littermate and transgenic animals at 11 weeks of age. We found no significant differences in median membrane potential (Down state) values between control and HD neurons $(t=1.42 ; \mathrm{df}=25 ; \mathrm{NS})$. No significant differences in the median Up state value were found between control and HD neurons $(t=0.98$; $\mathrm{df}=25 ; \mathrm{NS})$. Table 1 shows the median and median average deviation of the membrane potential values and input resistances of the neurons measured in this study.

To test if the pattern of fluctuations differed between control and HD neurons, we measured the distribution of durations of the Up and Down states. These distributions are plotted in the average dwell-time histograms shown in Figure 2 for Up states (Figure 2A) and Down states (Figure 2B). We found significant differences between the dwell-time distributions of the Down states between control and HD neurons, with the HD neurons having significantly more short down states $\left(\chi^{2}=25.13\right.$; $\left.\mathrm{df}=15 ; p \leq 0.05\right)$. This result indicates that the R6/2 neurons transition to the Up state more readily than do control neurons. No significant differences between the distributions of the Up state durations were found $\left(\chi^{2}=41.29 ; \mathrm{df}=51 ; \mathrm{NS}\right)$.

To test for serial dependence in the patterns of state durations, we measured the state durations as a function of the preceding state. Up and Down states were quantified using the method described in
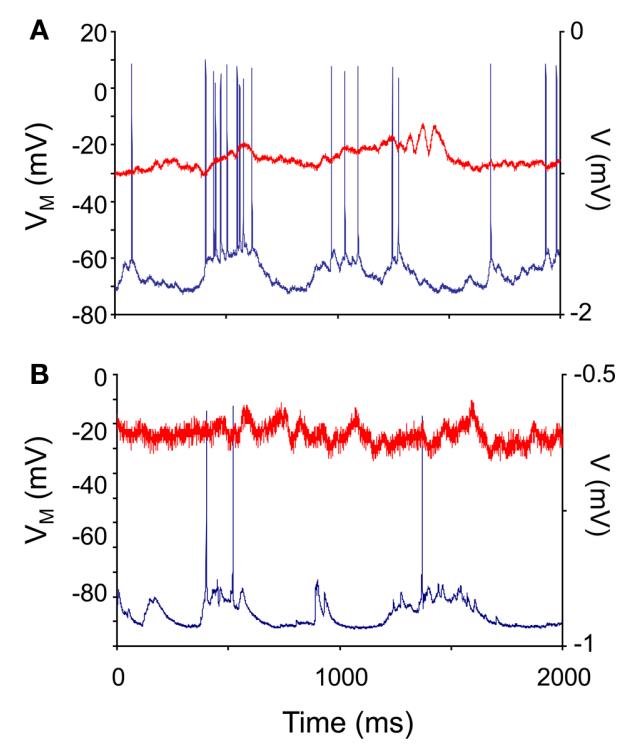

FIGURE 1 | Neocortical neurons in control and R6/2 model mice show spontaneous membrane potential fluctuations. In vivo intracellular recordings (blue traces) were taken from 10-week-old non-transgenic littermate control (A) and R6/2 (B) agranular neocortical neurons. All recorded neurons showed subthreshold fluctuations. The red traces are the electrocorticogram (ECoG) recorded simultaneously. 
Stern et al. (1997). Briefly, the all-points histogram of a 10-s sample of the membrane potential was plotted and fitted to a bimodal curve, separated by a single trough. A state transition was calculated as when the membrane voltage passed halfway from the trough to the state modal value. This method eliminates false state transitions. We measured the durations of the Down states as a function of the preceding Up state durations, the Up states as a function of the

Table 1 | Membrane potential and input resistance properties of mouse neocortical neuronal membrane potential and input resistance.

\begin{tabular}{lll}
\hline Parameter & Control & R6/2 \\
\hline$V_{\mathrm{M}}$ down & $-74 \pm 9 \mathrm{mV}(13)$ & $-78 \pm 12 \mathrm{mV}(13)$ \\
Up state depolarization & $11.5+4.5 \mathrm{mV}(13)$ & $13 \pm 6.5 \mathrm{mV}(13)$ \\
$R_{\text {in }}$ & $89.6+8.4 \mathrm{M} \Omega(13)$ & $65.1+9.2 \mathrm{M} \Omega(13)$
\end{tabular}

Numbers denote median \pm median absolute deviation. The numbers in parentheses denote the numbers of neurons in the group.
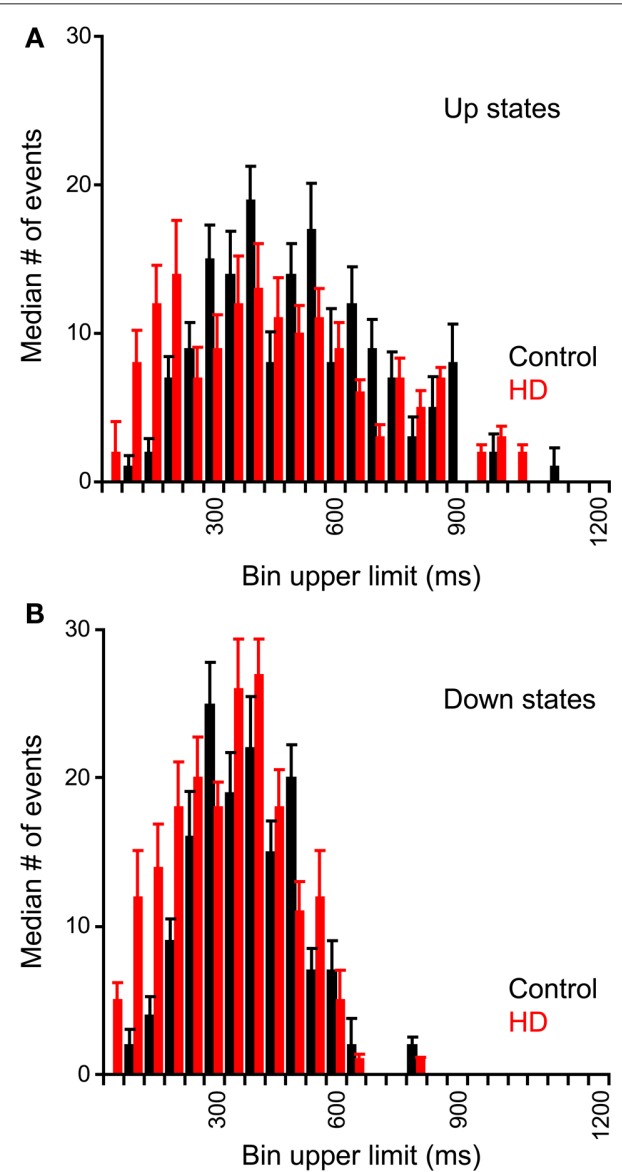

FIGURE 2 | Dwell-time histograms of Up and Down state durations of neocortical neurons. Durations of Up and Down states were measured from spontaneous recordings from non-transgenic littermate controls (black) and R6/2 (red) neocortical neurons. Average (median) histograms were constructed of Up States (A) and Down States (B). Error bars represent between-neuron median absolute deviations. For both Up and Down states, the HD distribution is shifted to the left; however, the differences between control and HD histograms are only significant for the Down states. preceding Down state durations, the Down states as a function of the preceding Down state durations, and the Up states as a function of the preceding Up state durations. Using the serial correlation test for renewal processes to test independence of successive intervals, no significant serial correlations were found between any successive state types of either population of neurons.

A logical place to expect changes in HD neocortical neurons is in the electrical properties of the neurons themselves, as a major neuropathological feature associated with the disease, the polyglutamine inclusions, are intracellular. One of the major changes in R6/2 neostriatal neurons found in acute slice neurons was a significant change in input resistance (Klapstein et al., 2001). We injected current pulses via the intracellular recording electrode to measure the membrane voltage changes and the excitation-to-frequency curves. Taken together, these are a measure of the input-output properties of the neurons. We injected $200 \mathrm{~ms}$ duration positive and negative current pulses of up to $\pm 1 \mathrm{nA}$, in steps of $100 \mathrm{pA}$. Each current step was repeated eight times. The average responses to such series are plotted in Figures $\mathbf{3 A , B}$, for a control and HD neuron, respectively.

It can be seen that the responses to current in the HD neurons are larger, indicating higher input resistance overall in these cells. Averages I/V plots for the neurons are shown in Figure 3C. The slopes of the curves are significantly different $(F=2.63 ; \mathrm{df}=25,25$; $p \leq 0.05$ ) indicating that the input resistances of the HD (red) mice are significantly greater than those of the controls (blue). Similar results were found in measurements in acute in vitro preparations of R6/2 neostriatal neurons (Klapstein et al., 2001; Ariano et al., 2005). However, because of the large differences in the amount of input between the in vivo and in vitro preparations, it was necessary to make this comparison in the intact brain to measure the functional consequences of the changes. The average excitation-tofrequency curves for the control (blue) and HD (red) neurons are plotted in Figure 3D. The most noticeable difference between the curves is the increased average responses to current pulses in the lower amplitude range of the HD neurons. In addition, it is clear that the average dynamic range of the neurons is reduced.

Given the hyperexcitability of the HD neocortical neurons shown by the average I/V and f/I plots in Figure 3, we hypothesized that their activity could be less synchronous with that of the neocortical network. The global coherence of the neocortical network is dependent on the envelope of input synchrony, as well as membrane properties of the neurons (Stern et al., 1997, 1998; Timofeev et al., 2000; Leger et al., 2005). We measured the global synchrony of the network by measuring coherence between the membrane potentials and the ECoG, using Hanning windows of $5 \mathrm{~s}$ with $50 \%$ overlap, following spike removal and downsampling to $1 \mathrm{kHz}$, giving a resolution of $0.2 \mathrm{~Hz}$. The Hanning window is an amplitude weighting of the time signal with the form $w=0.5 \times\left(1-\cos \left(2 \times \mathrm{pi} \times(1: \mathrm{M})^{\prime} /(\mathrm{M}+1)\right)\right)$; it is generally used for analysis of continuous signals to reduce the generation of side lobes in the frequency spectrum.

Harmonic means were taken of the coherences in each category Examples of single $5 \mathrm{~s}$ traces are shown in Figures 4A,B for control and HD neurons, respectively. The intracellular traces are plotted in blue, while the ECoG is plotted in red. The coherence averages are shown in Figure 4C for the control (black) and HD (red) neurons. 

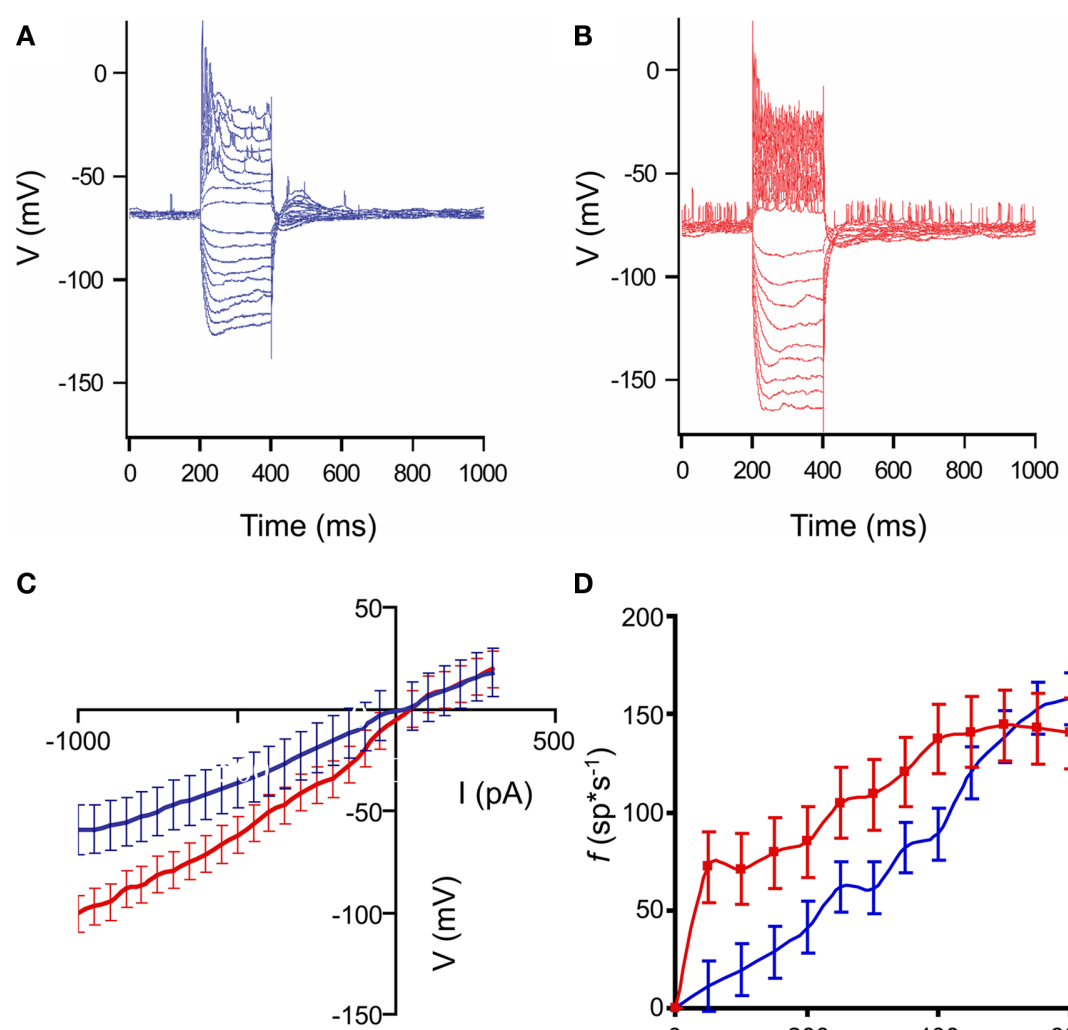

D

FIGURE 3 | Input-output characteristics of control and HD neurons. (A,B): Representative responses of neocortical neurons to intracellular current pulses. Each trace shown is an average response to eight stimuli. Pulses ranged from \pm 100 to $1000 \mathrm{pA}$ in amplitude, and $200 \mathrm{~ms}$ duration. (A): non-transgenic littermate control neuron; (B): R6/2 neuron. In the examples shown, the animals were 9 weeks of age. Note the increased voltage responses in the HD neuron, indicating higher input resistance, as well as the increased spiking, indicating

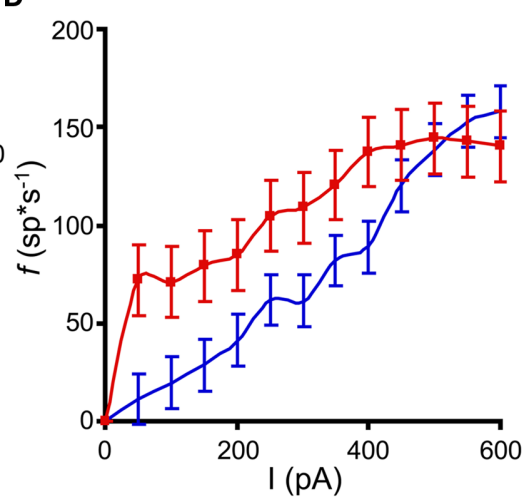

higher response frequency. (C): Average current-voltage plots of control (blue) and HD (red) neurons. Error bars represent between-neuron SD. Note the increased slope of the HD IN curve. (D). Average excitation-to-frequency ( $f / l)$ plots for control (blue) and HD (red) neurons. Error bars represent between-neuron SD. The lines are smoothed between the measured points. Note the increase in the $\mathrm{f} / \mathrm{l}$ slope in the lower part of the range, indicating that the HD neurons have smaller dynamic response ranges, and tend to respond abnormally to relatively weak stimuli.
The overall 95\% confidence intervals for the two spectra are shown by dashed lines (Rosenberg et al., 1989; Moran and Bar-Gad, 2010). We tested for differences in coherence in the low-frequency range by comparing the size of the peak at $<5 \mathrm{~Hz}$ over neurons between control and HD. This peak includes the energy in the range of the membrane potential state transitions, and was the only peak outside of the confidence intervals. We found that the coherence is significantly reduced in the HD animals (permutation test for independent samples; $t=2.71 ; \mathrm{df}=17 ; p \leq 0.05$ ), indicating that the state transitions of these neurons were significantly less correlated with the global activity than those of controls.

\section{DISCUSSION}

This study measured the functional properties of neocortical neurons in the R6/2 model of $\mathrm{HD}$ at ages when the polyglutamine inclusions are present in all such neurons. Although many in vitro studies of the electrophysiological changes of neostriatal and neocortical neurons have been published (Cepeda et al., 2001, 2003b, 2004, 2007; Klapstein et al., 2001; Laforet et al., 2001; Ariano et al., 2005; Andre et al., 2006, 2011; Cepeda and Levine, 2006; Joshi et al.,
2009), the functional changes in the corticostriatal input were not easily extrapolated from these studies, as the functional properties of neocortical neurons in vivo are different from those in vitro, since the Up state, dependent on large amounts of neocortical input, is not seen in acute reduced preparations.

It is of interest to observe that all neurons showed the subthreshold membrane potential dynamics seen in normal pyramidal neurons. This is also true in other disease models, such as Alzheimer's Disease (Stern et al., 2004). It is as if this type of activity is important enough to the neuron to be maintained as long as the neurons are alive. The principal changes found in the subthreshold membrane potential dynamics of the HD neurons was the increased number of shorter-duration Down states, indicating that the membrane potential of these cells transitioned more easily to the Up state, consistent with the increased input resistance and lower rectification measured in these neurons.

We found that the intrinsic properties of the HD neurons in vivo mirrored many of those observed in in vitro studies, Consistent with the results observed in neostriatal neurons, we found increased input resistance and reduced rectification properties in the neocortical 

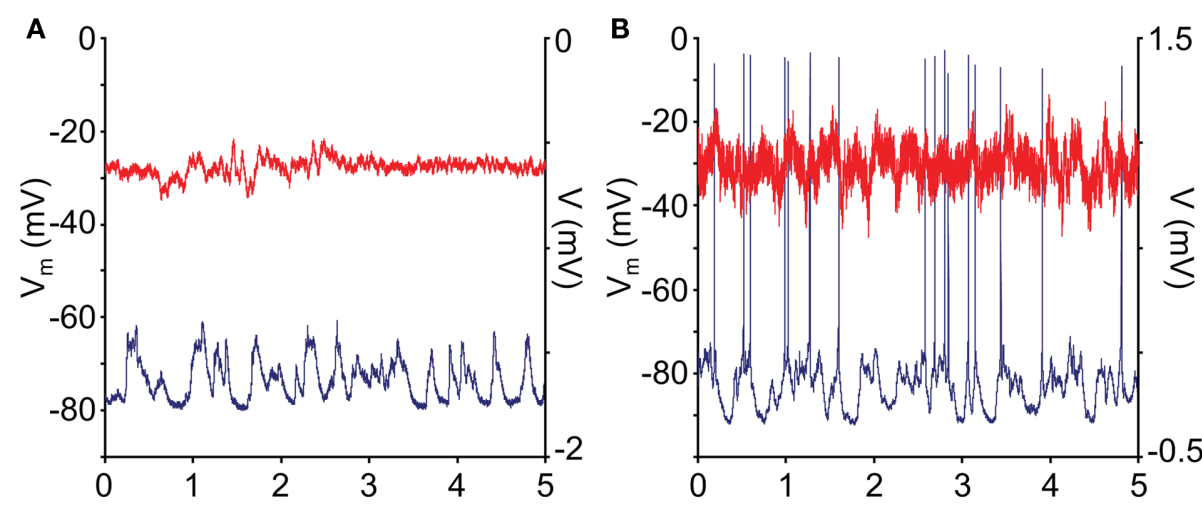

Time (s)

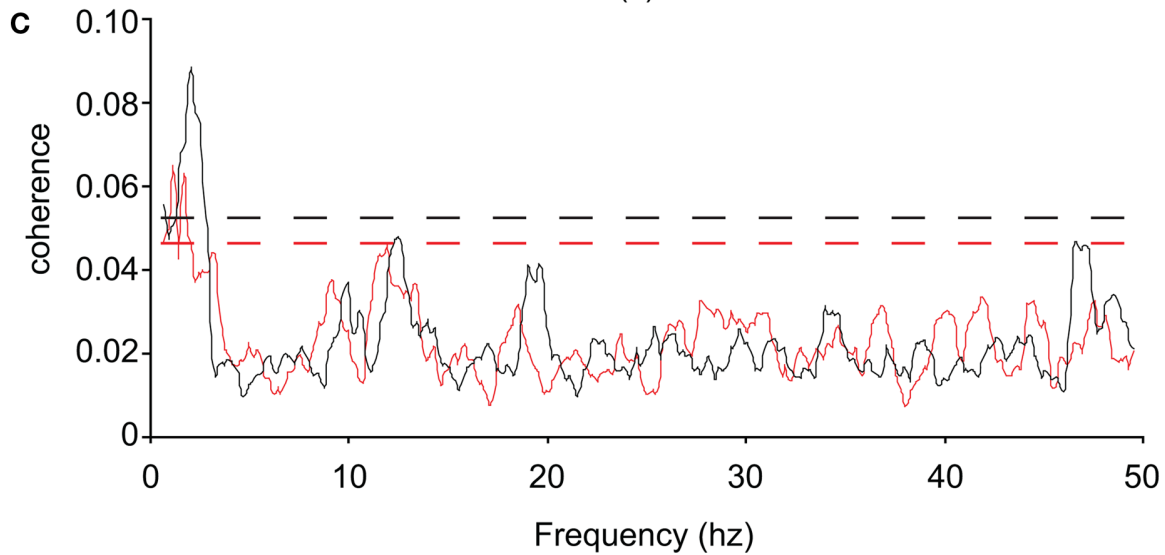

FIGURE 4 | Coherence of control and R6/2 neocortical activity. (A,B) Representative intracellular recordings (blue) and ECoG (red) from control and R6/2 mouse cortex. (C): average neuronal/neocortical coherence for the control (black trace) and HD (red trace) neurons. The coherence of the HD membrane potential with the global activity represented by the ECOG is significantly less than that of the controls in the low-frequency range $(<5 \mathrm{~Hz})$, which represents the state transition activity. The dashed lines represent the $95 \%$ confidence intervals of the spectra. neurons. Changes in rectification properties of HD neostriatal neurons (Ariano et al., 2005) and downregulation of potassium channel genes have been observed in previous studies (Zucker et al., 2005). It may be that a principal target of the accumulated polyglutamine is one or more families of potassium channels in the affected neocortical neurons as well, suggesting these channels as a potential therapeutic target.

A major finding of this study is the decreased dynamic range of the $f / I$ curves of the HD neurons. Given the reduced rectification properties of these neurons, it may be that the cells transition to the Up state more readily than do controls. This may give rise to increased activity of these neurons in awake animals (Walker et al., 2008), and may be the cause of the observed changes in corticostriatal post-synaptic currents observed in some studies (Cepeda et al., 2003a) In addition to changing the neocortical inputs, the functional consequences of these changes may be that the number of inputs to these neurons necessary to cause a transition from the Down state to the Up state is reduced, as are the number of inputs necessary to generate an action potential. The reduced membrane potential ECoG coherence of the HD neurons, shown in Figure 4, is further evidence that the membrane potentials of these neurons can transition for the Down to the Up states more easily than can controls.
The relative difficulty in eliciting the state transitions is a major determinant of neocortical function. Reducing the number of inputs necessary to evoke a state transition could give rise to severe functional abnormalities, such as affecting their ability participate in coordinated activity within neuronal assemblies. Our results could explain the abnormal correlated activity of neocortical and neostriatal neurons found in awake behaving $\mathrm{R} 6 / 2$ animals in which the synchrony of spikes is significantly reduced (Miller et al., 2008; Walker et al., 2008). In addition, the results observed could underlie some of the behavioral deficits observed in these animals such as reduction of coordinated activity (Carter et al., 1999).

Taken together, the results of this study demonstrate the functional consequences of the neocortical neuronal deficits observed in HD mouse models. While these deficits may underlie some of the changes observed in neostriatal neuronal activity in these models, it remains to be seen how much of those changes are due to the cellular changes resulting from HD pathology, and how much are due to the changes in neocortical input. The changes in neocortical input to neostriatal neurons coupled with differential changes in dopamine transmission (Andre et al., 2011) could explain the 
stereotypical behavior observed in these animals. This may be a major neuronal mechanism underlying the sensorimotor and cognitive symptoms of HD.

\section{CONCLUSION}

This study demonstrates a possible mechanism of the effect of cellular neuropathology on the properties of the neuronal network as a whole. Taken in conjunction with studies of the cellular effects of HD pathologies in in vitro preparations and studies in a wake,

\section{REFERENCES}

Andre, V. M., Cepeda, C., Fisher, Y. E., Huynh, M., Bardakjian, N., Singh, S., Yang, X. W., and Levine, M. S. (2011). Differential electrophysiological changes in striatal output neurons in Huntington's disease. J. Neurosci. 31, $1170-1182$.

Andre, V. M., Cepeda, C., Venegas, A., Gomez, Y., and Levine, M. S. (2006). Altered cortical glutamate receptor function in the R6/2 model of Huntington's disease. J. Neurophysiol. 95, 2108-2119.

Ariano, M. A., Cepeda, C., Calvert, C. R., Flores-Hernandez, J., HernandezEcheagaray, E., Klapstein, G. J., Chandler, S. H., Aronin, N., DiFiglia, M., and Levine, M. S. (2005). Striatal potassium channel dysfunction in Huntington's disease transgenic mice. J. Neurophysiol. 93, 2565-2574.

Bates, G. P., Mangiarini, L., Wanker, E. E., and Davies, S. W. (1998). Polyglutamine expansion and Huntington's disease. Biochem. Soc. Trans. 26, 471-475.

Carter, R. J., Lione, L. A., Humby, T., Mangiarini, L., Mahal, A., Bates, G. P., Dunnett, S. B., and Morton, A. J. (1999). Characterization of progressive motor deficits in mice transgenic for the human Huntington's disease mutation. J. Neurosci. 19, 3248-3257.

Cepeda, C., Ariano, M. A., Calvert, C. R., Flores-Hernandez, J., Chandler, S. H., Leavitt, B. R., Hayden, M. R., and Levine, M. S. (2001). NMDA receptor function in mouse models of Huntington disease. J. Neurosci. Res. $66,525-539$

Cepeda, C., Hurst, R. S., Calvert, C. R., Hernandez-Echeagaray, E., Nguyen, O. K., Jocoy, E., Christian, L. J., Ariano, M. A., and Levine, M. S. (2003a). Transient and progressive electrophysiological alterations in the corticostriatal pathway in a mouse model of Huntington's disease. J. Neurosci. 23, 961-969.

Cepeda, C., Hurst, R. S., FloresHernandez, J., Hernandez-Echeagaray, E., Klapstein, G. J., Boylan, M. K., Calvert, C. R., Jocoy, E. L., Nguyen, O. K., Andre, V. M., Vinters, H.V., Ariano,
M. A., Levine, M. S., and Mathern, G. W. (2003b). Morphological and electrophysiological characterization of abnormal cell types in pediatric cortical dysplasia. J. Neurosci. Res. 72 472-486.

Cepeda, C., and Levine, M. S. (2006). Dysfunctional channels are making noise in CAG triplet repeat disorders. Exp. Neurol. 202, 267-270.

Cepeda, C., Starling, A. J., Wu, N., Nguyen, O. K., Uzgil, B., Soda, T., Andre, V. M., Ariano, M. A., and Levine, M. S. (2004). Increased GABAergic function in mouse models of Huntington's disease: reversal by BDNF. J. Neurosci. Res. $78,855-867$.

Cepeda, C., Wu, N., Andre, V. M., Cummings, D. M., and Levine, M. S. (2007). The corticostriatal pathway in Huntington's disease. Prog. Neurobiol. 81, 253-271.

Cowan, R. L., and Wilson, C. J. (1994). Spontaneous firing patterns and axonal projections of single corticostriatal neurons in the rat medial agranular cortex. J. Neurophysiol. 71, 17-32.

Davies, S. W., Turmaine, M., Cozens, B. A., DiFiglia, M., Sharp, A. H., Ross, C. A., Scherzinger, E., Wanker, E. E., Mangiarini, L., and Bates, G. P. (1997). Formation of neuronal intranuclear inclusions underlies the neurological dysfunction in mice transgenic for the HD mutation. Cell 90, 537-548. S. W., Bates, G. P., Vonsattel, J. P., and huntingtin in neuronal intranuclear inclusions and dystrophic neurites in brain. Science 277, 1990-1993.

HDCRG. (1993). A novel gene containing a trinucleotide repeat that is expanded and unstable on Huntington's disease chromosomes. The Huntington's Disease Collaborative Research Group. Cell 72, 971-983.

Joshi, P. R., Wu, N. P., Andre, V. M., Cummings, D. M., Cepeda, C., Joyce, J. A., Carroll, J. B., Leavitt, B. R., Hayden, M. R., Levine, M. S., and Bamford, N. S. (2009). Age-dependent alterations of corticostriatal activity in the
DiFiglia, M., Sapp, E., Chase, K. O., Davies, Aronin, N. (1997). Aggregation of

behaving animals, intracellular recordings in vivo may provide a vital connection in understanding the effects of the neuropathology in $\mathrm{HD}$ on the function of the corticostriatal system.

\section{ACKNOWLEDGMENTS}

Supported by the Hereditary Disease Foundation and the Israel Science Foundation. The author Thanks Drs Jang-Ho Cha, Steven Hersch, and Michael Levine and Izhar Bar-Gad for useful suggestions, and Ms. Vered Kellner for help with the analysis.

YAC128 mouse model of Huntington disease. J. Neurosci. 29, 2414-2427.

Klapstein, G. J., Fisher, R. S., Zanjani, H., Cepeda, C., Jokel, E. S., Chesselet, M. F., and Levine, M. S. (2001) Electrophysiological and morphological changes in striatal spiny neurons in R6/2 Huntington's disease transgenic mice. J. Neurophysiol. 86, 2667-2677.

Laforet, G. A., Sapp, E., Chase, K. McIntyre, C., Boyce, F. M., Campbell, M., Cadigan, B. A., Warzecki, L., Tagle, D.A., Reddy, P.H., Cepeda, C., Calvert, C. R., Jokel, E. S., Klapstein, G. J. Ariano, M. A., Levine, M. S., DiFiglia, M., and Aronin, N. (2001). Changes in cortical and striatal neurons predict behavioral and electrophysiological abnormalities in a transgenic murine model of Huntington's disease. $J$ Neurosci. 21, 9112-9123.

Leger, J. F., Stern, E. A., Aertsen, A., and Heck, D. (2005). Synaptic integration in rat frontal cortex shaped by network activity. J. Neurophysiol. 93, 281-293.

Levine, M. S., Cepeda, C., Hickey, M. A., Fleming, S. M., and Chesselet, M. F. (2004). Genetic mouse models of Huntington's and Parkinson's diseases: illuminating but imperfect. Trends Neurosci. 27, 691-697.

Mangiarini, L., Sathasivam, K., Mahal, A. Mott, R., Seller, M., and Bates, G. P. (1997). Instability of highly expanded CAG repeats in mice transgenic for the Huntington's disease mutation. Nat. Genet. 15, 197-200.

Mangiarini, L., Sathasivam, K., Seller, M. Cozens, B., Harper, A., Hetherington, C., Lawton, M., Trottier, Y., Lehrach, H., Davies, S. W., and Bates, G. P. (1996). Exon 1 of the HD gene with an expanded CAG repeat is sufficient to cause a progressive neurological phenotype in transgenic mice. Cell 87, 493-506.

Miller, B. R., Walker, A. G., Shah, A. S., Barton, S. J., and Rebec, G. V. (2008). Dysregulated information processing by medium spiny neurons in striatum of freely behaving mouse models of Huntington's disease. J. Neurophysiol. 100, 2205-2216.

Moran, A., and Bar-Gad, I. (2010). Revealing neuronal functional organi- zation through the relation between multi-scale oscillatory extracellular signals. J. Neurosci. Methods 186, 116-129.

Rosenberg, J. R., Amjad, A. M., Breeze, P., Brillinger, D. R., and Halliday, D. M. (1989). The Fourier approach to the identification of functional coupling between neuronal spike trains. Prog. Biophys. Mol. Biol. 53, 1-31.

Sachdev, R. N., Ebner, F. F., and Wilson, C. J. (2004). Effect of subthreshold up and down states on the whiskerevoked response in somatosensory cortex. J. Neurophysiol. 92,3511-3521. Steriade, M., Nunez, A., and Amzica, F. (1993). A novel slow (<1 Hz) oscillation of neocortical neurons in vivo: depolarizing and hyperpolarizing components. J. Neurosci. 13, 3252-3265.

Stern, E. A., Bacskai, B. J., Hickey, G. A. Attenello, F. J., Lombardo, J. A., and Hyman, B. T. (2004). Cortical synaptic integration in vivo is disrupted by amyloid-beta plaques. J. Neurosci. 24 4535-4540.

Stern, E. A., Jaeger, D., and Wilson, C. J. (1998). Membrane potential synchrony of simultaneously recorded striatal spiny neurons in vivo. Nature 394, 475-478.

Stern, E. A., Kincaid, A. E., and Wilson, C. J. (1997). Spontaneous subthreshold membrane potential fluctuations and action potential variability of rat corticostriatal and striatal neurons in vivo. J. Neurophysiol. 77, 1697-1715.

Timofeev, I., Grenier, F., and Steriade, M. (2000). Impact of intrinsic properties and synaptic factors on the activity of neocortical networks in vivo. J. Physiol. Paris 94, 343-355.

Vonsattel, J. P., Myers, R. H., Stevens, T. J., Ferrante, R. J., Bird, E. D. and Richardson, E. P. Jr. (1985). Neuropathological classification of Huntington's disease. J. Neuropathol. Exp. Neurol. 44, 559-577.

Walker, A. G., Miller, B. R., Fritsch, J. N., Barton, S. J., and Rebec, G. V. (2008). Altered information processing in the prefrontal cortex of Huntington's disease mouse models. J. Neurosci. 28, 8973-8982. 
Zucker, B., Luthi-Carter, R., Kama, J. A., Dunah, A. W., Stern, E. A., Fox, J. H., Standaert, D. G., Young, A. B., and Augood, S. J. (2005). Transcriptional dysregulation in striatal projection and interneurons in a mouse model of Huntington's disease: neuronal selectivity and potential neuroprotec- tive role of HAP1. Hum. Mol. Genet. 14, 179-189.

Conflict of Interest Statement: The author declares that the research was conducted in the absence of any commercial or financial relationships that could be construed as a potential conflict of interest.
Received: 01 March 2011; accepted: 03 June 2011; published online: 16 June 2011. Citation: Stern EA (2011) Functional changes in neocortical activity in Huntington's disease model mice: an in vivo intracellular study. Front. Syst. Neurosci. 5:47. doi: 10.3389/ fnsys.2011.00047
Copyright $\odot 2011$ Stern. This is an openaccess article subject to a non-exclusive license between the authors and Frontiers Media SA, which permits use, distribution, and reproduction in other forums, provided the original authors and source are credited and other Frontiers conditions are complied with. 